\title{
Fractal analysis of nuclear histology integrates tumor and stromal features into a single prognostic factor of the oral cancer microenvironment
}

Pinaki Bose ${ }^{1,2}$, Nigel T Brockton ${ }^{3}$, Kelly Guggisberg ${ }^{4}$, Steven C Nakoneshny ${ }^{5}$, Elizabeth Kornaga ${ }^{6}$, Alexander C Klimowicz ${ }^{7}$ Mauro Tambasco ${ }^{8^{*}}$ and Joseph C Dort ${ }^{5^{*}}$

\begin{abstract}
Background: The lack of prognostic biomarkers in oral squamous cell carcinoma (OSCC) has hampered treatment decision making and survival in OSCC remains poor. Histopathological features are used for prognostication in OSCC and, although useful for predicting risk, manual assessment of histopathology is subjective and labour intensive. In this study, we propose a method that integrates multiple histopathological features of the tumor microenvironment into a single, digital pathology-based biomarker using nuclear fractal dimension (nFD) analysis.

Methods: One hundred and seven consecutive OSCC patients diagnosed between 1998 and 2006 in Calgary, Canada were included in the study. nFD scores were generated from DAPI-stained images of tissue microarray (TMA) cores. Ki67 protein expression was measured in the tumor using fluorescence immunohistochemistry (IHC) and automated quantitative analysis (AQUA ${ }^{\oplus}$ ). Lymphocytic infiltration (LI) was measured in the stroma from haematoxylin-eosin (H\&E)-stained TMA slides by a pathologist.

Results: Twenty-five (23.4\%) and 82 (76.6\%) patients were classified as high and low nFD, respectively. nFD was significantly associated with pathological tumor-stage ( $p T$-stage; $P=0.01)$ and radiation treatment $(\mathrm{RT} ; P=0.01)$. High nFD of the total tumor microenvironment (stroma plus tumor) was significantly associated with improved disease-specific survival (DSS; $P=0.002)$. No association with DSS was observed when nFD of either the tumor or the stroma was measured separately. pT-stage $(P=0.01)$, pathological node status (pN-status; $P=0.02$ ) and RT $(P=0.03)$ were also significantly associated with DSS. In multivariate analysis, nFD remained significantly associated with DSS [HR $0.12(95 \% \mathrm{Cl} 0.02-0.89, P=0.04)]$ in a model adjusted for pT-stage, pN-status and RT. We also found that high nFD was significantly associated with high tumor proliferation $(P<0.0001)$ and high $\mathrm{LI}(P<0.0001)$, factors that we and others have shown to be associated with improved survival in OSCC.

Conclusions: We provide evidence that nFD analysis integrates known prognostic factors from the tumor microenvironment, such as proliferation and immune infiltration, into a single digital pathology-based biomarker. Prospective validation of our results could establish nFD as a valuable tool for clinical decision making in OSCC.
\end{abstract}

\footnotetext{
* Correspondence: mtambasco@mail.sdsu.edu; jdort@ucalgary.ca

${ }^{8}$ Department of Physics, San Diego State University, San Diego, California 92182-1233, USA

${ }^{5}$ Department of Surgery, Division of Otolaryngology-Head and Neck Surgery, University of Calgary, Calgary, Alberta T2N 4Z6, Canada

Full list of author information is available at the end of the article
} 


\section{Background}

Almost 30000 individuals are diagnosed with oral squamous cell carcinoma (OSCC) each year in North America and approximately 6000 of these patients succumb to the disease, annually [1]. OSCC is an aggressive disease and even favourable treatment outcomes are associated with significant morbidity. Five-year survival rates for OSCC have remained between 40 and 50\% for the past several decades. Biomarkers that can identify aggressive disease at diagnosis and inform treatment decisions might improve survival outcomes and quality of life for OSCC patients. Although several prognostic markers for OSCC have been described in the literature, treatment is directed predominantly by the tumor-node-metastasis (TNM) staging system.

The tumor microenviroment is a dynamically interacting entity composed of tumor cells and the surrounding stroma. These interactions are not only critical for tumor growth and progression but also for treatment sensitivity/ resistance. Therefore, effective prognostic biomarkers should ideally incorporate features of both tumor and stroma, leading to a more comprehensive assessment of tumor biology. Histopathological features have been previously used for prognostication in OSCC. BrandweinGensler and colleagues described a histologic risk assessment score based on pattern of invasion (POI), perineural invasion (PNI) and lymphocytic infiltration (LI) $[2,3]$. These authors propose that achieving negative resection margins do not guarantee local disease-free and overall survival benefits. On the other hand, a combination of histopathological features of the tumor (POI and PNI) and stroma (LI) accurately predicted risk of local recurrence and survival. Although the manual assessment of histological features is a powerful technique for predicting risk, it requires expert subject knowledge of head and neck histopathology and can be very labour intensive. Since few diagnostic laboratories have access to specialized head and neck pathologists, integration of histological feature analysis into a single, digital histopathology-based biomarker may improve the utility and encourage clinical adoption of this type of prognostic testing.

Fractal dimension (FD) is a mathematical measure of the irregularity and complexity of a shape and may be used for the digital assessment and quantification of histological features in the tumor microenvironment [4]. In contrast to our intuitive notion of dimension (i.e. the topological dimension), which is an integer value (0 for a point, 1 for a line, 2 for a plane, etc.), the FD can be a non-integer value that is greater than the topological dimension. The extent to which the FD of an object may be greater than the topological dimension depends on the space filling capacity of the object. FD is a noninteger number that quantifies the degree of space filling of an object. True mathematical fractals exhibit a higher degree of space filling because they exhibit exact or statistical self-similarity in structural patterns when examined to infinitely small scales. As such, actual fractals do not exist in nature, since there is a fundamental natural limitation to the scaling behaviour of natural objects [5]. However, FD analysis has found widespread use in medical image analysis because it lends itself naturally to the pragmatic characterization of irregular non-Euclidean structures found in medical images [6,7]. One such application of FD has been to discriminate the architectural complexity of biological structures associated with neoplastic states. Previous studies have applied FD analysis for the diagnosis, staging and prognosis of several cancer-types including breast $[4,8]$, prostate [9,10], colon [11], lung [12], endometrial [13], gall bladder [14], larynx [15] and OSCC [16]. FD analysis of nuclear histology digitally quantifies the space filling properties of nuclei. Such analysis, when performed on the entire tumor microenvironment (tumor and stroma) can be a source of rich prognostic information.

We have previously used fluorescence immunohistochemistry (IHC) and automated quantitative analysis $\left(\mathrm{AQUA}^{\circ}\right)$ to investigate the prognostic value of proteins associated with apoptosis $[17,18]$, proliferation [19] and hypoxia $[20,21]$ in OSCC. We have also reported that the prognostic impact of these biomarkers differs according to their cellular distribution within the tumor microenvironment. Tissue microarrays (TMAs) used to examine protein biomarkers are ideally-suited for digital histological analysis since TMA cores contain both tumor and stromal tissue compartments. When performing $\mathrm{AQUA}^{\circ}$, images of each whole TMA core are generated and the nuclei are routinely co-stained with 4',6-diamidino-2-phenylindole (DAPI) to differentiate nuclear/cytoplasmic localization of a biomarker. In this study, we computed the fractal properties of DAPIstained nuclei in whole TMA cores. We hypothesized that nFD analysis will integrate tumor and stromal characteristics commonly incorporated in OSCC histopathological risk assessment methods into a single, prognostic factor for OSCC. We report that nFD is a robust and powerful independent prognosticator of patient outcome that integrates the proliferative properties of the tumor compartment and the immunologic properties of the stromal compartment into a unified prognostic entity that is amenable to clinical translation.

\section{Methods}

\section{Patient cohort}

This study conforms to the Tri-council Policy Statement for Research with Human Subjects (Canada) and was approved by the University of Calgary Conjoint Health Research Ethics Board. Our retrospective study cohort consisted of 107 histologically confirmed treatment 
naïve, surgically resected OSCC patients diagnosed between 1998 and 2006 at the Foothills Medical Centre, Calgary, Canada. Eligible patients had no prior history of head and neck cancer. Patients received post-operative radiotherapy based on the presence of metastatic lymph nodes, extra-capsular spread or positive surgical margins. Clinico-pathological characteristics of the patient cohort are described in Table 1.

\section{Tissue microarray (TMA) construction}

Archived formalin-fixed paraffin-embedded (FFPE) tumor blocks were retrieved for TMA construction. Haematoxylineosin (H\&E)-stained slides were reviewed by the study pathologist (KG) to select blocks with sufficient tumor content. For each patient included in the study, three $0.6 \mathrm{~mm}$ cores were randomly sampled from the tumour-bearing areas of selected FFPE block using a Beecher Manual Tissue Microarrayer (Beecher Instruments Inc. WI, USA). Approximately 100 patients (each with triplicate cores) were included on a TMA block. Slides were assembled using $4 \mu \mathrm{m}$ thick sections from the TMA block.

\section{Fractal dimension analysis}

TMAs were immunofluorescently stained as previously described [18]. High resolution images of nuclei, defined by positive DAPI-stained regions, for each TMA core were collected for subsequent analysis as part the automated quantitative analysis $\left(\mathrm{AQUA}^{\circ}\right)$ process. Images were acquired at 20X magnification corresponding to a resolution of $0.468 \mu \mathrm{m} /$ pixel and saved in tagged image file format (.tiff) for fractal analysis. Cores were excluded from analysis if they were out of focus, tissue was folded, or there was insufficient tumor present (less than 100 tumor cells).

We applied an automated fractal analysis technique that we developed in previous work $[5,10]$ to quantify the degree of space filling of nuclei. In summary, this technique involves the following steps:

1. Application of a series of intensity thresholds to convert the acquired grey-scale DAPI images (from $\mathrm{AQUA}^{\circ}$ ) into a series of binary images to derive the outlines of nuclei.

2. Application of the box counting method (with appropriate spatial scale range for our structures of interest - nominally $\sim 4$ to $60 \mu \mathrm{m})[5,10]$ to compute the fractal dimension of each outline image obtained from step 3.

3. Identification of the global maximum from a plot of fractal dimension versus intensity threshold. This maximum corresponds to the fractal dimension of the pathological structures [10].

Our automated fractal analysis method was applied to a total of 321 TMA cores (3 cores for each of the 107
Table 1 Clinico-pathological characteristics of the patient cohort

\begin{tabular}{|c|c|c|c|c|c|c|}
\hline & $\begin{array}{l}\text { \# Of } \\
\text { cases }\end{array}$ & (\%) & $\begin{array}{l}\text { nFD } \\
\text { low }^{\text {a }}\end{array}$ & $\begin{array}{l}\text { nFD } \\
\text { high }^{\text {a }}\end{array}$ & $\begin{array}{l}\mathrm{FE} \\
P \text { - value }\end{array}$ & $\begin{array}{l}\text { DSS } \\
\text { (LR } P \text { - value) }\end{array}$ \\
\hline $\begin{array}{l}\text { Number of } \\
\text { events }\end{array}$ & - & - & 33 & 1 & - & - \\
\hline Gender & & & & & 0.81 & 0.457 \\
\hline Male & 70 & 65.42 & 53 & 17 & & \\
\hline Female & 37 & 34.58 & 29 & 8 & & \\
\hline Age & 107 & NA & $61.1^{\mathrm{b}}$ & $59.8^{b}$ & 0.68 & nd \\
\hline pT-Stage & & & & & 0.01 & 0.01 \\
\hline pT1/pT2 (low) & 61 & 57.01 & 41 & 20 & & \\
\hline pT3/pT4 (high) & 44 & 41.12 & 40 & 4 & & \\
\hline Missing & 2 & 1.87 & 1 & 1 & & \\
\hline pN-status & & & & & 0.25 & 0.02 \\
\hline pNO & 66 & 61.68 & 48 & 18 & & \\
\hline $\mathrm{pN} 1 / \mathrm{pN} 2$ & 41 & 38.32 & 34 & 7 & & \\
\hline $\begin{array}{l}\text { Smoking } \\
\text { history }\end{array}$ & & & & & 0.60 & 0.27 \\
\hline Never & 28 & 26.168 & 20 & 8 & & \\
\hline Ever & 78 & 72.897 & 61 & 17 & & \\
\hline Missing & 1 & 0.935 & 1 & 0 & & \\
\hline $\begin{array}{l}\text { Alcohol } \\
\text { history }\end{array}$ & & & & & 0.43 & 0.14 \\
\hline Never & 11 & 10.28 & 10 & 1 & & \\
\hline Ever & 57 & 53.27 & 43 & 14 & & \\
\hline Missing & 39 & 36.45 & 29 & 10 & & \\
\hline $\begin{array}{l}\text { Tumor } \\
\text { differentiation }\end{array}$ & & & & & 0.23 & 0.18 \\
\hline Well & 15 & 14.02 & 10 & 5 & & \\
\hline Moderate & 56 & 52.34 & 47 & 9 & & \\
\hline Poor & 12 & 11.21 & 9 & 3 & & \\
\hline Missing & 24 & 22.43 & 16 & 8 & & \\
\hline Treatment & & & & & 0.01 & 0.03 \\
\hline Surgery & 34 & 31.78 & 20 & 14 & & \\
\hline Surgery + RT & 73 & 68.22 & 62 & 11 & & \\
\hline \multicolumn{7}{|l|}{ Ki67 } \\
\hline Low & 43 & 76.19 & 40 & 3 & 0.001 & 0.002 \\
\hline High & 62 & 23.81 & 40 & 22 & & \\
\hline \multicolumn{7}{|l|}{ LI } \\
\hline Low & 53 & 50.47 & 48 & 5 & 0.001 & 0.002 \\
\hline High & 52 & 49.43 & 32 & 20 & & \\
\hline
\end{tabular}

${ }^{a}$ Cut-point was determined using $\mathrm{X}$-tile.

${ }^{\mathrm{b}}$ Mean age.

nFD: nuclear fractal dimension; $\mathrm{pT}$-stage: pathological T-stage; $\mathrm{pN}$ status: pathological node status; RT: radiotherapy; FE: Fisher's exact; LR: logrank, LI: lymphocytic infiltration. Significant $P$ - values are shown in BOLD.

patient samples), and for each patient the mean nFD from the three TMA cores was used in statistical analyses. 


\section{Ki67 fluorescence IHC}

Ki67 staining has been described previously [19]. Briefly, TMAs were stained for DAPI (Invitrogen), Ki67 (mouse monoclonal, clone MIB1, DAKO) and Pan-cytokeratin (PCK; guinea pig polyclonal, ACRIS). We used the Aperio Scanscope ${ }^{\bullet}$ FL slide scanner for automated fluorescent image acquisition and HistoRx AQUAnalysis ${ }^{\circ}$ software version 2.3.4.1 for automated image analysis.

\section{Lymphocytic infiltration}

H\&E stained OSCC TMA cores were evaluated for the presence of LI at the invasive boundary of the tumor at 200X microscope magnification by the study pathologist (KG). All mononuclear cells including lymphocytes and plasma cells were scored (granulocytes and other polymorphonuclear leukocytes were excluded). Necrotic areas near the invasive tumor boundaries were excluded from LI assessment. LI was classified as a four-tiered variable: zero infiltration, weak infiltration, intermediate infiltration and strong infiltration. For each patient, the maximum value of the LI among three TMA cores was used for statistical analyses.

\section{Statistical analysis}

$\mathrm{X}$-Tile version 3.6.1 software was used to determine optimal cut-points to dichotomize continuous nFD scores [22]. In Table 1, Fisher's exact test was used to compare clinical covariates between the two patient groups defined by low or high nFD. Kaplan-Meier curves and Cox proportional hazards models were used to assess association with 5-year disease-specific survival (DSS). Clinical covariates that are usually associated with prognosis in OSCC such as pathological T-stage (pT-stage) and pathological node status (pN-status) were subjected to Cox univariate analysis. Clinical covariates that were significantly associated with DSS in univariate analysis were included in a multivariate model with nFD. In all analyses, a $P$-value of $<0.05$ was considered statistically significant. All statistical analyses were performed using Stata 13 data analysis and statistical software (StataCorP LP, College Station, Tx, USA).

\section{Results}

\section{Cohort characteristics}

Our study was conducted and reported according to Reporting recommendations for tumor marker prognostic studies (REMARK) criteria for reporting tumor biomarker prognostic studies [23]. The median age at diagnosis for the study cohort was 62.35 years (range: 25.73 - 95.12 years). Median survival was 77.85 months; survivor follow-up duration ranged between 1.3 and 156.9 months [standard deviation $=33.3$ ]. All patients were treated with primary surgery and $73(68.2 \%)$ received post-operative radiotherapy. Univariate analyses of the association of clinico-pathological variables with DSS are presented in Table 1. pT-stage $(P=0.01)$, pN-status $(P=0.02)$ and treatment (whether patients received post-operative radiotherapy; $P=0.03$ ) were significantly associated with DSS. Patients with high nFD did not differ significantly from patients with low $\mathrm{nFD}$ in terms of age of diagnosis, gender, $\mathrm{pN}$-status, smoking history, alcohol history and tumor differentiation status (Fisher's exact test).

\section{Fractal analysis of nuclei and survival analyses}

nFD scores ranged between 1.19 and 1.84, with a median of 1.52, lower quartile 1.42, and upper quartile 1.64. Figure 1 shows representative monochromatic DAPIstained images of TMA cores with low (1.28), intermediate (1.47) and high (1.84) nFD. All TMA cores examined contained nuclei from both the tumor and the stromal tissue compartments. Twenty-five (23.4\%) patients were classified as high nFD and $82(76.6 \%)$ were classified as low nFD. Among the clinical covariates assessed, high pT-stage was significantly associated with low nFD scores (Fisher's Exact $P=0.01$; Table 1). Also, most patients who received post-operative radiotherapy had low nFD scores (Fisher's Exact $P=0.01$; Table 1 ). In our entire cohort of 107 OSCC patients, high nFD was associated with significantly better DSS compared to low $\mathrm{nFD}$ (Figure 2A); the HR estimate was 0.09 (95\% CI, 0.01 to 0.64 ), reflecting a $91 \%$ reduction in DSS nonachievement in patients with high nFD $(P=0.02$; Table 2). A significant association between nFD and DSS was also observed when the analysis was restricted to patients who received post-operative radiotherapy (73 patients; logrank $P=0.01$; Figure $2 \mathrm{~B}$ ); no association between nFD and DSS was observed in patients who were treated with surgery alone (logrank $P=0.26$; Figure $2 \mathrm{C}$ ). Furthermore, nFD remained an independent prognostic factor in our OSCC cohort [HR 0.11 (95\% CI, 0.02 to $0.83), P=0.03$ ] after adjusting for other known prognostic factors including pT-stage [HR 2.26 (95\% CI 1.10 to 4.50, $P=0.02)$ ] and pN-status [HR 2.14 (95\% CI 1.10 to $4.30, P=0.03$ )] (Table 2). The nFD of the tumor compartment or stromal compartment alone were not significantly associated with survival (data not shown).

\section{Association between nFD and tumor proliferation}

We have previously reported that increased tumor cell proliferation in OSCC is associated with significantly better survival that may be attributed to an improved response to post-operative radiotherapy [19]. Tumor proliferation was assessed by Ki67 staining of nuclei in the PCK-stained tumor compartment. Figure 3A shows representative images of DAPI-stained nuclei from TMA cores within the PCK-stained tumor compartment and corresponding H\&E-stained slides from the same patient. The 

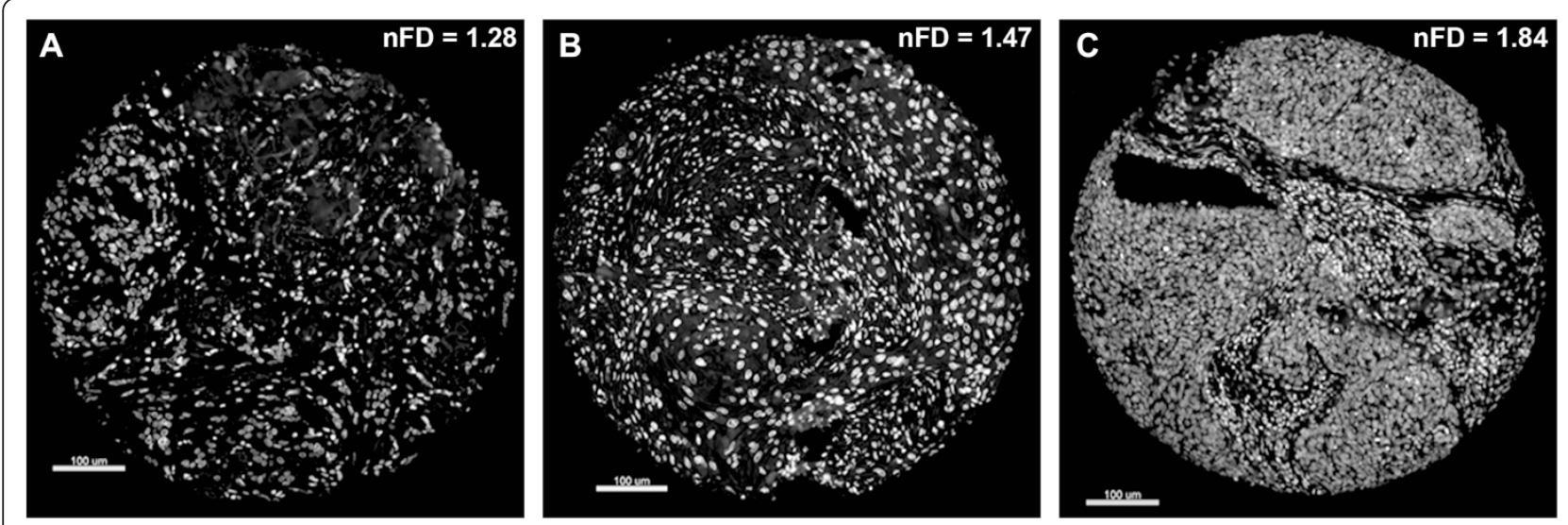

Figure 1 Representative DAPI-stained images of individual tissue microarray (TMA) cores used as substrates for nuclear fractal dimension (nFD) analysis. Image of an entire TMA core with (A) low nFD, (B) intermediate nFD and (C) high nFD.

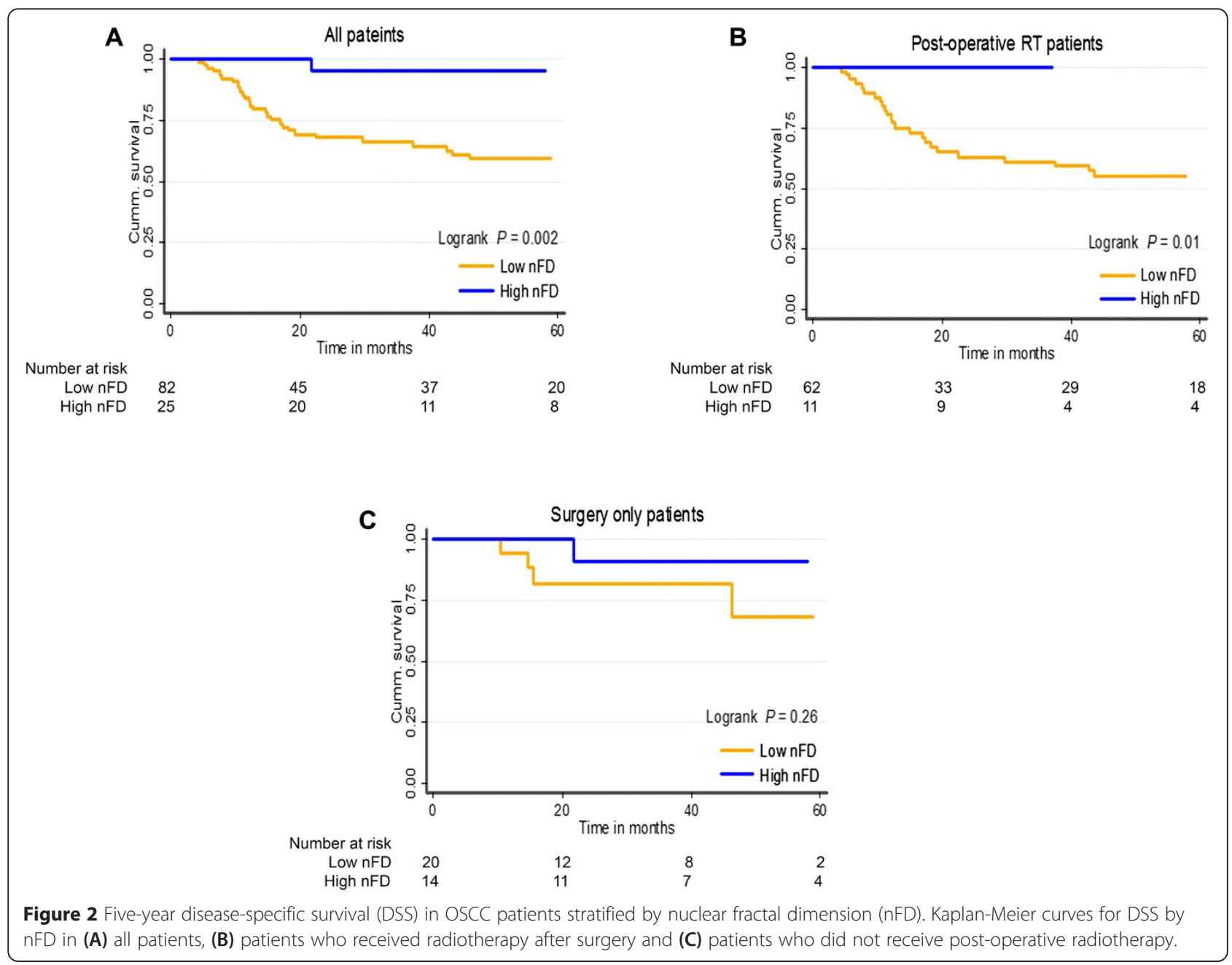


Table 2 Univariate and multivariate analysis of 5-Year Disease-Specific Survival (DSS)

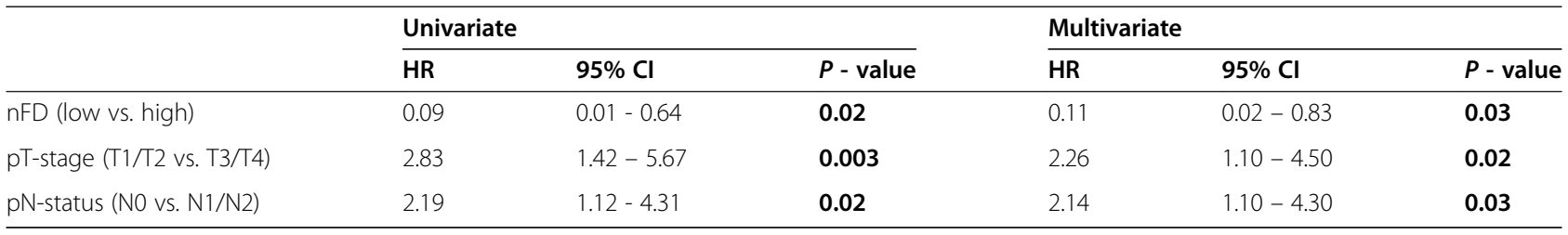

HR: hazard ratio; Cl: confidence interval. HRs estimated from stratification of Cox proportional hazard models. Significant $P$ - values are shown in BOLD.

box and whisker plots (Figure 3B) illustrate that the mean nFD was significantly higher in the high proliferative index group $(P<0.0001)$.

\section{Association between nFD and LI}

In order to better understand how nFD is correlated with characteristics of the tumor microenvironment, we studied the association between nFD and stromal LI. Figure 3C shows representative fluorescent images of DAPI-stained nuclei from TMA cores in the PCK-negative stromal compartment with corresponding nFD scores (upper panel) and H\&E-stained TMA cores from the same patients showing LI (lower panel). Considerable heterogeneity in terms of LI scores was observed among TMA cores from the same patient, ranging from weak infiltration in one core to strong infiltration in another. The core showing the maximum infiltration was used as the representative core for each patient. As evident from the box and whisker plots (Figure 3D), nFD values were positively correlated with increased LI in the stroma $(P<0.0001)$. In
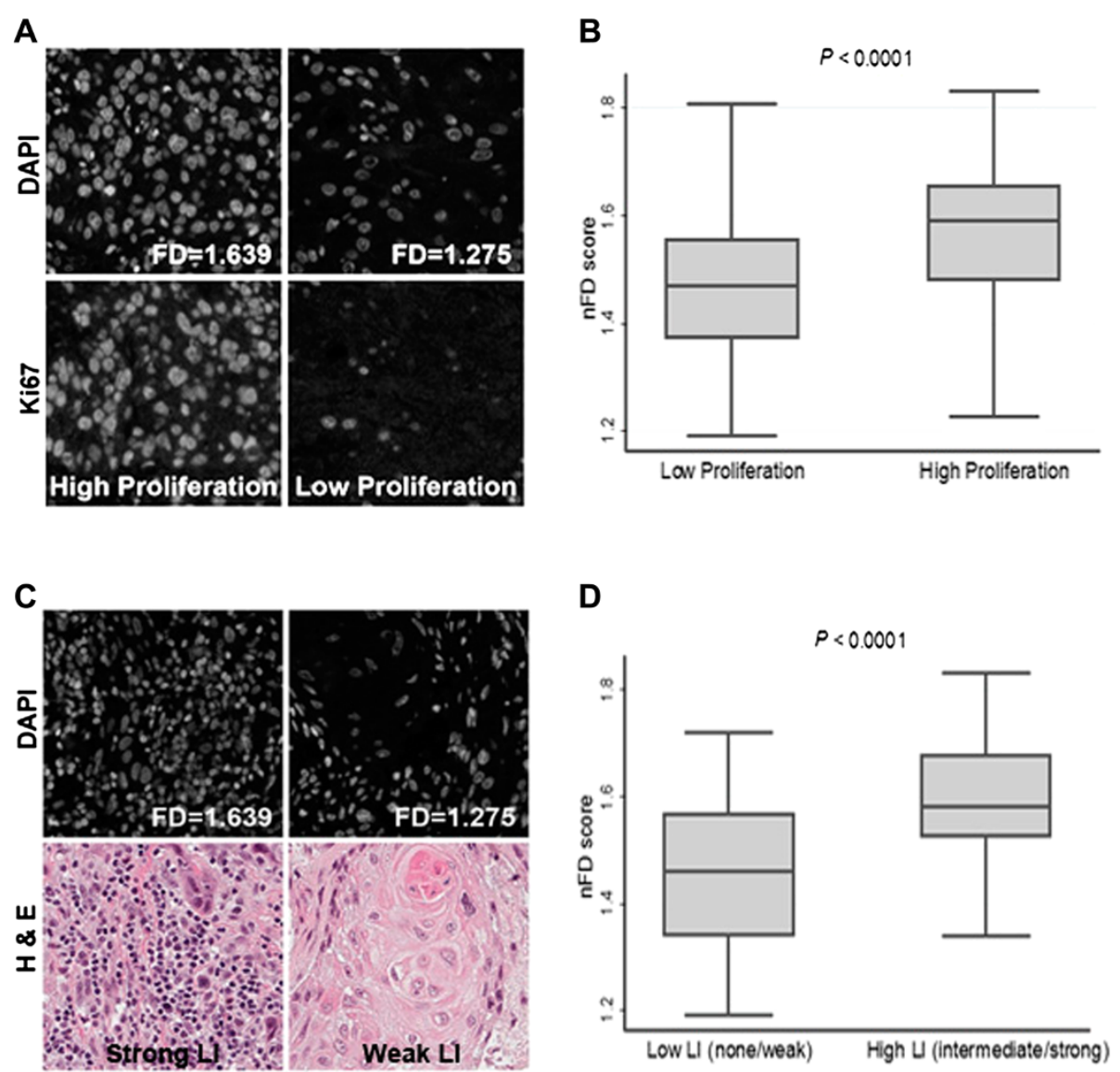

Figure 3 Association between nuclear fractal dimension (nFD) and features of the tumor microenvironment. (A) Representative DAPI-stained images of TMA cores with high and low nFD (upper panels) and images of the same cores stained for Ki67 (lower panels). (B) Box and whisker plot showing the association between nFD and tumor proliferation. (C) Representative DAPI-stained images of TMA cores with high and low nFD (upper panels) and $\mathrm{H}$ \& E-stained images of cores from the same patient that were used for assessing lymphocytic infiltration (Ll; lower panels). (D) Box and whisker plot showing the association between $\mathrm{nFD}$ and LI. 
agreement with previous reports [24-26], high LI was associated with significantly improved survival in our OSCC cohort ( $P=0.001$; Additional file 1: Figure S1).

\section{Discussion}

We report a digital histopathologal image-based prognostic biomarker (nFD) derived from fractal analysis of DAPI-stained nuclei. This single measure integrates features of both the stromal and tumor compartments in the tumor microenvironment. nFD can effectively discriminate between OSCC patients with good and worse prognosis and was an independent prognostic indicator in our OSCC cohort when the model was adjusted for established prognostic clinical covariates. A strong positive correlation was observed between $\mathrm{nFD}$ and a pathologistscored assessment of LI in the stroma. nFD was also positively correlated with proliferation (scored using fluorescence $\mathrm{IHC}$ and $\mathrm{AQUA}{ }^{\circ}$ ), an important tumor-associated prognostic marker. The significance of both these features, independently, suggests that nFD scores are an effective method for the automated, image-based, integration of both stromal and tumor features with acknowledged prognostic value.

OSCC is a serious public health problem worldwide and the lack of effective prognostic biomarkers adversely affects patient management and survival outcomes. This has led researchers to look beyond the traditional TNM staging system and investigate biological correlates to histopathological features of the tumor microenvironment. Staining with DAPI is a routine component of IHC that helps identify nuclei. We hypothesized that FD analysis of DAPI-stained images (computer-acquired when performing AQUAnalysis ${ }^{\circ}$ ), could provide a digital histology-based prognostic factor for OSCC that might be more objective and less labour-intensive than traditional histopathological analysis. FD analysis has been previously used in OSCC. Several researchers have demonstrated that FD can discriminate between normal versus malignant oral tissue $[27,28]$. Goutzanis and colleagues have used FD to assess vascularization and also $\mathrm{nFD}$ as a prognostic factor [16,29]. However, contrary to our results, these authors report that high nFD is associated with poor prognosis [16]. It is worth noting that almost all previously reported studies have used 3,3'dichlorobenzidine (DAB) IHC-based images for FD analysis. Also, these studies did not take into account the tumor microenvironment that might provide valuable biologic information relevant to prognosis. DAPI-based nuclear staining is more robust than DAB IHC-based techniques since it is not affected by antibody specificity issues. Also DAPI staining is relatively easy to perform that protein-based IHC since protein is more sensitive than DNA to pre-analytical variables, particularly when the motive is to preserve overall DNA structure rather than specific base pairs. Also, DAPI staining allows for multiplexing of diverse stains, allowing for staining of additional proteins that can, e.g. discriminate the tumor (PCK) and stromal (vimentin) compartments [21]. Interestingly, we found that nFD scores from the stromal or tumor compartment alone did not show a significant association with survival. However, a robust association with survival was observed when nFD from the whole tissue core (tumor plus stroma) was considered.

In order to understand how well digital nFD-based histopathological analysis correlates with expert pathological assessment of stromal morphological parameters associated with survival, we compared LI, scored by a pathologist, with $\mathrm{nFD}$ scores. We also studied the relationship between $\mathrm{nFD}$ and tumor proliferation in order to evaluate if nFD correlated with tumor-associated prognostic features. LI has been previously reported to be associated with prognosis in OSCC [24-26] and was significantly associated with both DSS and OS in our OSCC cohort as well (Additional file 1: Figure S1). We observed that high nFD was associated with increased LI in the stroma and cell proliferation in the tumor. We believe that high nFD tumor and stroma might reflect increased proliferation in the tumor and the presence of infiltrating immune cells in the stroma. Both have been shown to be associated with improved prognosis attributed to increased susceptibility to radiotherapy. We found that patients with high nFD scores have significantly better survival when treated with post-operative radiotherapy compared to patients with low nFD (Figure 3b). Although further investigation is required, nFD analysis may represent a summary measure incorporating tumor proliferation and the immune involvement that predicts response to radiotherapy [30]. Therefore, high nFD might represent a state where high proliferation renders tumor cells sensitive to radiotherapy and dying cells are cleared by the infiltrating immune cells, creating a "perfect storm" for the tumor.

\section{Conclusions}

The histopathological scoring system proposed by Brandwein-Gensler et al. is a powerful tool but its broad implementation may be limited by its labour intensiveness and the requirement for extensive training. Additionally, consistency of scoring is a substantial risk given the high degree of inter-observer variability in other oral histopathology-based systems, among pathologists [31,32]. Digital pathology-based nFD scoring incorporates multiple biomarkers and therefore might provide a more reliable and objective indicator of prognosis compared to single biomarker-based assays. We believe that a comprehensive approach to the analysis of tumor microenvironment, such as the one presented here, will improve prognostication and outcomes in OSCC. 


\section{Consent}

Written informed consent was obtained from the patient for the publication of this report and any accompanying images.

\section{Additional file}

Additional file 1: Figure S1. Five-year disease-specific survival (DSS) in OSCC patients stratified by lymphocytic infiltration (LI). Kaplan-Meier curves for DSS in patients stratified by high and low (LI).

\section{Abbreviations}

AQUA: Automated quantitative analysis; Cl: Confidence interval; DAB: 3,3'dichlorobenzidine; DAPI: 4',6-diamidino-2-phenylindole; DSS: Disease-specific survival; FFPE: Formalin-fixed, paraffin-embedded; H\&E: Haematoxylin-eosin; HR: Hazard ratio; IHC: Immunohistochemistry; LI: Lymphocytic infiltration; nFD: Nuclear fractal dimension; OSCC: Oral squamous cell carcinoma; PCK: Pan-cytokeratin; PNI: Perineural invasion; POI: Pattern of invasion; pN-status: Pathological node status; pT-stage: Pathological T-stage; REMARK: Reporting recommendations for tumor marker prognostic studies; RT: Radiation treatment; TMA: Tissue microarray; TNM: Tumor-node-metastasis.

\section{Competing interests}

This study was funded by the Ohlson Research Initiative, which functions within the Faculty of Medicine at the University of Calgary. The corresponding author (JCD) is the Director of the Ohlson Research Initiative. This does not alter our adherence to all BMC Cancer policies as detailed in the guide for authors. All other authors declare that they have no competing interests.

\section{Authors' contributions}

PB designed and coordinated the study and drafted the manuscript. NTB contributed to study design and drafting of the manuscript. KG was the designated study pathologist. PB and SCN performed statistical analyses. EK performed the fluorescence $\mathrm{IHC}$ and AQUAnalysis. ACK contributed to study design. MT and JCD conceived the study, supervised statistical analyses and helped with manuscript editing. JCD also contributed patients to the study. All authors read and approved the final manuscript.

\section{Authors' information}

PB is presently a postdoctoral fellow (PDF) at the British Columbia Cancer Agency Genome Sciences Centre (BCGSC). A part of the present study was conducted while PB was a PDF at the Ohlson Research Inititative (ORI) in Head and neck cancer, University of Calgary. NTB is a molecular cancer epidemiologist and research scientist with Cancer Epidemiology \& Prevention Research, CancerControl Alberta, Alberta Health Services. KG is an anatomic pathologist with Calgary Laboratory Services and is also the President of the Alberta Society of Laboratory Physicians. SCN is a research assistant and lead project manager at the ORI. EK is a Quality and Data Coordinator with the Translational Laboratories Functional Tissue Imaging Unit (FTIU) at the Tom Baker Cancer Centre, Alberta Health Services. ACK is a Principal Scientist in Immunology and Inflammation Research, Boehringer Ingelheim Pharmaceuticals. MT is an Associate Professor and a boardcertified medical physicist at the Department of Physics, San Diego State University. JCD is Chief - Otolaryngology, Head \& Neck Surgery - Foothills Medical Centre and Program Leader - Head \& Neck Surgical Oncology Program, Cumming School of Medicine, University of Calgary. He also serves as the Executive Director of the ORI.

\section{Acknowledgements}

We would like to thank Dr. Don Morris for his support of the Functional Tissue Imaging Unit (FTIU) as Director of the Translational Laboratories, Tom Baker Cancer Centre. This work was supported by funding from the Ohlson Research Initiative.

\section{Author details}

'Department of Oncology, University of Calgary, Calgary, Canada. ${ }^{2}$ Current Address: Canada's Michael Smith Genome Sciences Centre, British Columbia
Cancer Agency, Vancouver, British Columbia, Canada. ${ }^{3}$ Department of Cancer Epidemiology and Prevention Research, CancerControl Alberta, Alberta Health Services, Calgary, Alberta T2N 2T9, Canada. ${ }^{4}$ Department of Anatomic Pathology, Calgary Laboratory Services, Rockyview General Hospital, Calgary, Alberta T2V 1P9, Canada. ${ }^{5}$ Department of Surgery, Division of Otolaryngology-Head and Neck Surgery, University of Calgary, Calgary, Alberta T2N 4Z6, Canada. ${ }^{6}$ Functional Tissue Imaging Unit, Translational Laboratories, Tom Baker Cancer Centre, Calgary, Alberta T2N 4N2, Canada. ${ }^{7}$ Immunology and Inflammation Research, Boehringer Ingelheim Pharmaceuticals, Inc, Ridgefield, Connecticut 06877, USA. ${ }^{8}$ Department of Physics, San Diego State University, San Diego, California 92182-1233, USA

\section{Received: 21 December 2014 Accepted: 28 April 2015}

Published online: 15 May 2015

\section{References}

1. GLOBOCAN 2012: Estimated cancer incidence, mortality and prevalence worldwide in 2012. [http://globocan.iarc.fr/Default.aspx]

2. Brandwein-Gensler M, Teixeira MS, Lewis CM, Lee B, Rolnitzky L, Hille JJ, et al. Oral squamous cell carcinoma: histologic risk assessment, but not margin status, is strongly predictive of local disease-free and overall survival. Am J Surg Pathol. 2005;29:167-78.

3. Brandwein-Gensler M, Smith RV, Wang B, Penner C, Theilken A, Broughel D, et al. Validation of the histologic risk model in a new cohort of patients with head and neck squamous cell carcinoma. Am J Surg Pathol. 2010;34:676-88.

4. Tambasco M, Eliasziw M, Magliocco AM. Morphologic complexity of epithelial architecture for predicting invasive breast cancer survival. J Transl Med. 2010:8:140.

5. Braverman B, Tambasco M. Scale-specific multifractal medical image analysis Comput Math Methods Med. 2013;2013:262931.

6. Heymans O, Fissette J, Vico P, Blacher S, Masset D, Brouers F. Is fractal geometry useful in medicine and biomedical sciences? Med Hypotheses. 2000;54:360-6.

7. Lopes R, Betrouni N. Fractal and multifractal analysis: a review. Med Image Anal. 2009;13:634-49.

8. Tambasco M, Magliocco AM. Relationship between tumor grade and computed architectural complexity in breast cancer specimens. Hum Pathol. 2008:39:740-6.

9. Tabesh A, Teverovskiy M, Pang HY, Kumar VP, Verbel D, Kotsianti A, et al. Multifeature prostate cancer diagnosis and Gleason grading of histological images. IEEE Trans Med Imaging. 2007;26:1366-78.

10. Tambasco M, Costello BM, Kouznetsov A, Yau A, Magliocco AM. Quantifying the architectural complexity of microscopic images of histology specimens. Micron. 2009:40:486-94.

11. Esgiar AN, Naguib RN, Sharif BS, Bennett MK, Murray A. Fractal analysis in the detection of colonic cancer images. IEEE Trans Inf Technol Biomed. 2002;6:54-8.

12. Oczeretko E, Juczewska M, Kasacka I. Fractal geometric analysis of lung cancer angiogenic patterns. Folia Histochem Cytobiol. 2001;39 Suppl 2:75-6.

13. Dey P, Rajesh L. Fractal dimension in endometrial carcinoma. Anal Quant Cytol Histol. 2004;26:113-6.

14. Waliszewski P. Distribution of gland-like structures in human gallbladder adenocarcinomas possesses fractal dimension. J Surg Oncol. 1999;71:189-95.

15. Delides A, Panayiotides I, Alegakis A, Kyroudi A, Banis C, Pavlaki A, et al. Fractal dimension as a prognostic factor for laryngeal carcinoma. Anticancer Res. 2005;25:2141-4.

16. Goutzanis L, Papadogeorgakis N, Pavlopoulos PM, Katti K, Petsinis V, Plochoras I, et al. Nuclear fractal dimension as a prognostic factor in oral squamous cell carcinoma. Oral Oncol. 2008;44:345-53.

17. Bose P, Klimowicz AC, Kornaga E, Petrillo SK, Matthews TW, Chandarana S, et al. Bax expression measured by AQUAnalysis is an independent prognostic marker in oral squamous cell carcinoma. BMC Cancer. 2012;12:332

18. Bose P, Thakur SS, Brockton NT, Klimowicz AC, Kornaga E, Nakoneshny SC, et al. Tumor cell apoptosis mediated by cytoplasmic ING1 is associated with improved survival in oral squamous cell carcinoma patients. Oncotarget. 2014:5:3210-9.

19. Klimowicz AC, Bose P, Nakoneshny SC, Dean M, Huang L, Chandarana S, et al. Basal Ki67 expression measured by digital image analysis is optimal for 
prognostication in oral squamous cell carcinoma. Eur J Cancer. 2012:48:2166-74.

20. Brockton NT, Klimowicz AC, Bose P, Petrillo SK, Konno M, Rudmik L, et al High stromal carbonic anhydrase IX expression is associated with nodal metastasis and decreased survival in patients with surgically-treated oral cavity squamous cell carcinoma. Oral Oncol. 2012;48:615-22.

21. Klimowicz AC, Bose P, Petrillo SK, Magliocco AM, Dort JC, Brockton NT. The prognostic impact of a combined carbonic anhydrase IX and Ki67 signature in oral squamous cell carcinoma. Br J Cancer. 2013;109:1859-66.

22. Camp RL, Dolled-Filhart M, Rimm DL. X-tile: a new bio-informatics tool for biomarker assessment and outcome-based cut-point optimization. Clin Cancer Res. 2004;10:7252-9.

23. McShane LM, Altman DG, Sauerbrei W, Taube SE, Gion M, Clark GM, et al. Reporting recommendations for tumor marker prognostic studies. J Clin Oncol. 2005;23:9067-72.

24. Carrillo JF, Carrillo LC, Cano A, Ramirez-Ortega MC, Chanona JG, Aviles A, et al. A Retrospective cohort study of prognostic factors in patients with squamous cell carcinoma of the oral cavity and oropharynx. Head Neck. 2014. doi:10.1002/hed.23914

25. Jing J, Li L, He W, Sun G. Prognostic predictors of squamous cell carcinoma of the buccal mucosa with negative surgical margins. J Oral Maxillofac Surg. 2006:64:896-901.

26. Ogawa Y, Nishioka A, Hamada N, Terashima M, Inomata T, Yoshida S, et al. Immunohistochemical study of c-fos-positive lymphocytes infiltrated into human squamous cell carcinomas of the head and neck during radiation therapy and its clinical significance. Clin Cancer Res. 1997;3:2301-7.

27. Klatt J, Gerich CE, Grobe A, Opitz J, Schreiber J, Hanken H, et al. Fractal dimension of time-resolved autofluorescence discriminates tumour from healthy tissues in the oral cavity. J Craniomaxillofac Surg. 2014;42:852-4.

28. Landini G, Rippin JW. Fractal dimensions of the epithelial-connective tissue interfaces in premalignant and malignant epithelial lesions of the floor of the mouth. Anal Quant Cytol Histol. 1993;15:144-9.

29. Goutzanis LP, Papadogeorgakis N, Pavlopoulos PM, Petsinis V, Plochoras I,

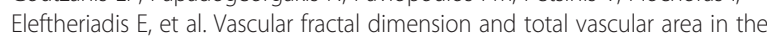
study of oral cancer. Head Neck. 2009;31:298-307.

30. Balermpas P, Michel Y, Wagenblast J, Seitz O, Weiss C, Rodel F, et al. Tumour-infiltrating lymphocytes predict response to definitive chemoradiotherapy in head and neck cancer. Br J Cancer. 2014;110:501-9.

31. Kujan O, Khattab A, Oliver RJ, Roberts SA, Thakker N, Sloan P. Why oral histopathology suffers inter-observer variability on grading oral epithelial dysplasia: an attempt to understand the sources of variation. Oral Oncol. 2007:43:224-31.

32. Fischer DJ, Epstein JB, Morton TH, Schwartz SM. Interobserver reliability in the histopathologic diagnosis of oral pre-malignant and malignant lesions. J Oral Pathol Med. 2004;33:65-70.

\section{Submit your next manuscript to BioMed Central and take full advantage of:}

- Convenient online submission

- Thorough peer review

- No space constraints or color figure charges

- Immediate publication on acceptance

- Inclusion in PubMed, CAS, Scopus and Google Scholar

- Research which is freely available for redistribution

Submit your manuscript at www.biomedcentral.com/submit 